\title{
Separation of the Pyro- and Piezoelectric Response of Electroactive Polymers for Sensor Applications
}

\author{
H. F. Castro ${ }^{1, a}$, S. Lanceros-Mendez ${ }^{2, b}$ and J. G. Rocha ${ }^{1, c}$ \\ ${ }^{1}$ University of Minho, Industrial Electronics, Portugal \\ ${ }^{2}$ University of Minho, Physics, Portugal \\ ahelderfilipe@sapo.pt, ${ }^{\mathrm{b}}$ lanceros@fisica.uminho.pt, ${ }^{\text {'}}$ gerardo@dei.uminho.pt
}

Keywords: Electroactive Polymers; Piezoelectricity; Pyroelectricity; PVDF.

\begin{abstract}
One of the main problems for the use of ferroelectric materials for sensor applications, especially for tactile sensors, is the separation of the pyro- and piezoelectric responseproduced by temperature and pressure changes, respectively. We present two different approaches to achieve this goal. The first approach is based on the study of the signal in the frequency domain and uses a signal processing to separate the piezoelectric response from the pyroelectric one. The second approach is based on a double layer configuration of electroactive polymers. In this case, the piezoelectric response is equal in each layer, whereas, the pyroelectric response dominates in the layer exposed to the temperature variations. The pyro- and piezoelectric responses are separated by estimating the difference between the signals of two layers and the signal from one of the layers, correspondingly.
\end{abstract}

\section{Introduction}

Piezoelectric and pyroelectric effects are widely used in the field of sensors and actuators [1, 2]. Many piezoelectric materials also have pyroelectric properties. Further, all pyroelectric materials show piezoelectric response. Synthetic organic polymers containing molecular dipoles in which alignment can be achieved by applying an electrical field (DC) and which produces polarization variation under applied stress exhibit the aforementioned pyro- and piezoelectric properties and are interesting for applications because of its flexibility and easy fabrication of piezo-active films. The organic polymer PVDF is one of the most studied polymer systems that exhibit ferroelectric, pyroelectric and piezoelectric properties $[3,4]$.

This polymer has great potential as a tactile sensor $[5,6]$ especially if the piezoelectric and pyroelectric response, i.e. pressure and temperature records are separated $[1,7]$.

All piezoelectric materials develop an electrical charge produced by mechanical stress. Decay of the electrical charges is determined by the dielectric constant and the internal resistance of the piezoactive film, as well as the input impedance of the interface electronics to which this film is connected $[1,7]$.

The piezoelectric charge (strain) coefficients predict, for small stress (or strain) levels, the surface charge density originated by external stress. In the charge mode and under conditions approaching a short circuit, the generated charge density is given by Eq.1.

$D=\frac{Q}{A}=d_{3 n} X_{n} \quad(n=1,2,3)$

The mechanical axis $n$ of the applied stress (or strain), by convention, is 1 for length (or stretch) direction, 2 for width (or transverse) direction and 3 for thickness direction. Here $D$ is the surface charge density developed, $Q$ the charge developed, $A$ the conductive electrode area, $d_{3 n}$ the appropriate piezoelectric coefficient for the axis of applied stress or strain and $X_{n}$ the stress applied in the relevant direction.

In the voltage mode, the open-circuit output voltage is given by Eq. 2. 


$$
V_{0}=g_{3 n} X_{n} t, \quad(\mathrm{n}=1,2,3)
$$

Where $g_{3 n}$ is the appropriate piezoelectric coefficient for the axis of applied stress, $X_{n}$ is the applied stress in the $n$-th direction and $t$ the film thickness.

Pyroelectric materials develop and electric polarization if their temperature is changed and are necessarily piezoelectric.

As these materials absorb thermal energy, they expand or contract, thereby inducing secondary piezoelectric signals. As piezo film is heated, the dipoles within the film exhibit random motion by thermal agitation. These causes a reduction in the average polarization of the film, generating a charge build up on the film surfaces. The output current is proportional to the rate of temperature change $\Delta T$. Electrical charge $Q$ and voltage produced by temperature increase (or decrease) in a given films of area $\mathrm{A}$ and thickness $t$ and permittivity $\varepsilon$ is determined by the pyroelectric coefficient, $\rho$, Eq. 3 [7].

$\left\{\begin{array}{l}Q=\rho \Delta T A \\ V=\frac{\rho t \Delta T}{\varepsilon}\end{array}\right.$

This article explores the separation of pyro- and piezoelectic electrical response in the signal of a sensor based on ferroelectric $\beta$-PVDF polymer. To accomplish this objective the mutual piezo and pyroelectric response emerged in the signal of a single layer sensor is recorded and analysed in the frequency /time domains by means of a signal processing that allows the separation of the signals. The second approach is based on a bilayer arrangement of $\beta$-PVDF films. In this case the piezoelectric signal produced by each layer is similar whereas the pyroelectric signal differs in such a manner that the external layer of PVDF film is more sensitive to temperature variations. These facts allow a simpler separation of the piezo and pyroelectric responses.

\section{Experimental set up}

Commercially available $28 \mu \mathrm{m}$ thick poled $\beta$-PVDF (Measurement Specialties Inc.) was electroded by aluminum thermal evaporation. Single and double layer sensors are shown in Figure 1.

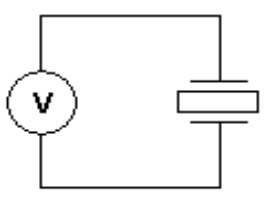

(a)

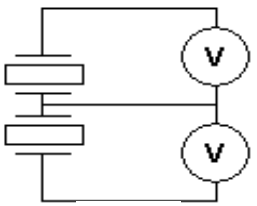

(b)

Fig. 1: a) Single and b) double layer sensors measurement circuit.

The signals produced by the applied pressure (piezoelectric signal) and temperature variation (pyroelectric signal) were measured with a Tektronix TDS 303 4B oscilloscope. The pressure and the temperature variations were applied and controlled with home-made setups.

\section{Results and Discussion}

Figure 2 shows the voltage produced by a single piezoelectric polymer layer, when a pressure impulse is applied. The maximal response is obtained with a $3 \mathrm{~ms}$ delay, though the overall response of the single layer sensor extends up to $45 \mathrm{~ms}$.

In order to check the pyroelectric response of the same single piezoelectric polymer layer, the voltage produced by a temperature variation of $5^{\circ} \mathrm{C}$ in $10 \mathrm{~s}$ was recorded as shown in and analysed, 
Figure 3. The radiation source consists of an aluminum plate heated up to $100^{\circ} \mathrm{C}$. The polymer layer was placed $2 \mathrm{~cm}$ above the radiation source and the temperature at its surface is $38^{\circ} \mathrm{C}$. In this case a slower variation of the induced voltage is obtained.

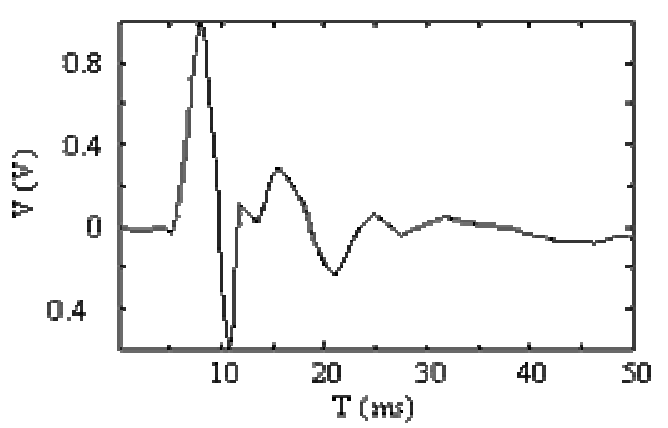

Fig. 2: Voltage produced by a single piezoelectric polymer layer, when a pressure impulse is applied.

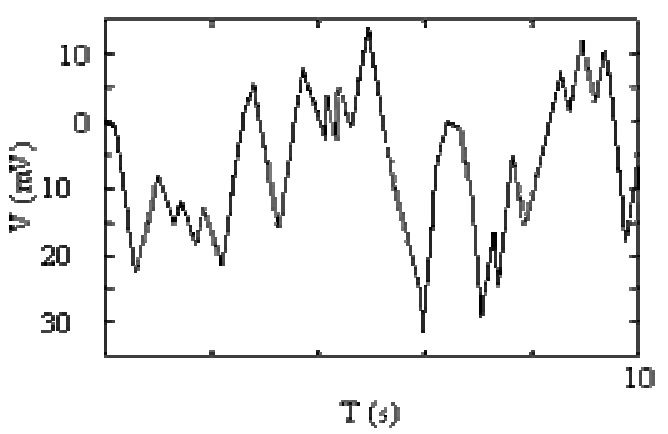

Fig. 3: Voltage produced by a single piezoelectric polymer layer, when placed at $2 \mathrm{~cm}$ from a $100^{\circ} \mathrm{C}$ hot plate. The temperature at the polymer surface is $38^{\circ} \mathrm{C}$.

Figures 4 and 5 show the spectral response of signals shown in Figures 1 and 2 calculated by applying the FFT transform. The spectral response of the material when pressure is applied extends up to $300 \mathrm{~Hz}$. The temperature response, on the other hand, extends up to $2 \mathrm{~Hz}$. Due to the different spectral bandwidth of the signals, the separation of the pressure and temperature response is possible by means of filters.

In order to record only the piezoelectric response a band pass filter should be tuned to $200 \mathrm{~Hz}$ (central frequency) with a bandwidth of $200 \mathrm{~Hz}$. Thereby only the $100 \mathrm{~Hz}$ to $300 \mathrm{~Hz}$ signals will be present on the filter output. On the other hand, to obtain the pyroelectric response, a low pass filter should be used, that allows output signals from DC up to $2 \mathrm{~Hz}$.

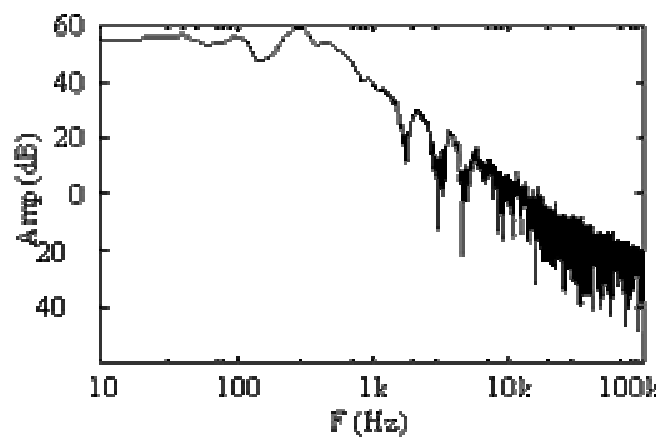

Fig. 4: Spectral response of a single piezoelectric polymer layer, when a pressure impulse is applied. 


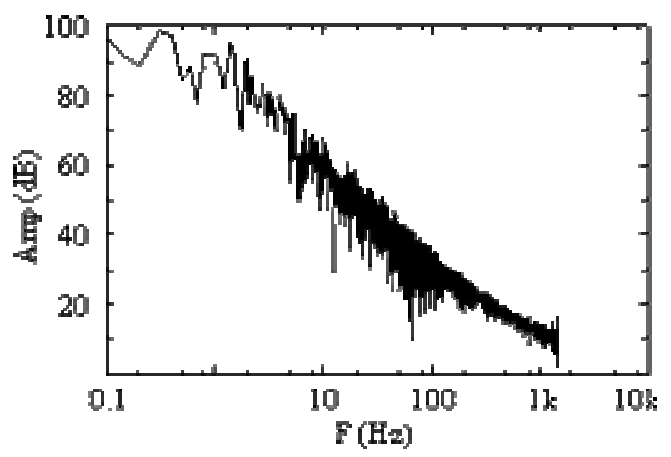

Fig. 5: Spectral response of a single piezoelectric polymer layer, when placed at 2 $\mathrm{cm}$ from a $100^{\circ} \mathrm{C}$ hot plate. The temperature at the polymer surface is $38^{\circ} \mathrm{C}$.

In the following the results of a bilayer arrangement will be presented. Figure 6 shows the voltages produced by two superimposed layers, when a pressure impulse is applied. The piezoelectric voltage response, when the force is applied to the upper layer, is similar for both layers excepting for a phase shift of approximated $0.5 \mathrm{~ms}$.

For the pyroelectric signal of the bilayer, Figure 7 shows the voltage produced when the sensor is placed at $2 \mathrm{~cm}$ from a $100^{\circ} \mathrm{C}$ hot plate. The temperature at the top polymer surface is $38^{\circ} \mathrm{C}$. In this case, whereas the layer receiving the radiation suffers variations consistent to the signal measured for a single layer sensor, the second layer shows two times smaller amplitude variations. Thus, in the case of a double layer configuration, the piezoelectic and pyroelectric responses can be independently recorded as follows: whereas the piezoelectric signal can be directly obtained from the lower layer, the pyroelectric signal will be obtained by subtracting the signals of the two layers.

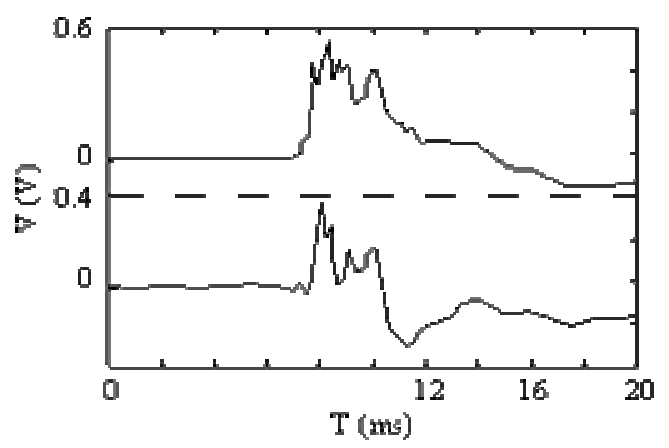

Fig. 6: Voltages produced by two superimposed piezoelectric polymer layers, when a pressure impulse is applied (above: upper layer; bellow: lower layer).

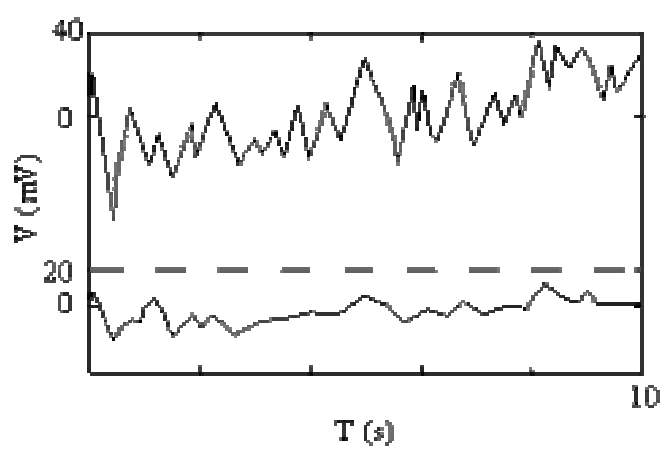

Fig. 7: Voltage produced by two superimposed piezoelectric polymer layers, when placed at $2 \mathrm{~cm}$ from a $100{ }^{\circ} \mathrm{C}$ hot plate. The temperature at the polymer surface receiving the heat is $38^{\circ} \mathrm{C}$. 


\section{Conclusion}

Two measurement methods using single and double arrangements of polymer layers were investigated. Both methods allow the separation of the piezoelectric and pyroelectric response. In the case of a single layer arrangement, more electronics and more complex, with filters tuned at different frequencies, is required in order to separate both signals.

In the case of a double layer sensor, the sensor preparation is more complex but the electronic is simpler: the pyroelectric effect is obtained from the difference between the signal from the two layers and the piezoelectric effect can be directly obtained from the lower layer.

\section{Acknowledgements}

Financial support from FCT (Grants POCTI/CTM/33501/99 and POCI/CTM/59425/2004). Samples provided by Measurement Specialties Inc.

\section{References}

[1] J. Fraden, "Handbook for Modern Sensors", AIP, New York, (1997) and references therein

[2] H. Singh Nalwa, J. Macromol. Chem. Phys., C31(4), (2001) pp. 341-417.

[3] T. Furukawa, Phase Transitions Vol 18 (1989), p. 143

[4] E. Fukada, "History and Recent Progress in Piezoelectric Polymers", IEEE Transactions on Ultrasonics, Ferroelectrics, and Frequency Control, 47, 6, (2000), pp.1227-1290, , and references therein.

[5] R.R. Reston, E.S. Kolesar, "Robotic tactile sensor array fabricated from a piezoelectric polyvinylidene fluoride film, in Proceedings of the IEEE NAECON, (1990), pp. 1139-1144.

[6] J. Engel, J. Chen, Z. Fan, C. Liu, Polymer micromachined multimodal tactile sensor, Sensors and Actuators A: Physical, 117 (1), (2005) pp. 50-61,.

[7] Piezo Film Sensors Technical Manual, Measurements Specialties, (1999) 